\title{
Correction to: Formulas of Absolute Moments
}

\author{
Gwo Dong Lin and Chin-Yuan Hu \\ Academia Sinica, Taipei, Taiwan
}

\section{Correction to: Sankhyā A https://doi.org/10.1007/s13171-019-00196-x}

After publication of this paper, it was found out that there are still corrections which were not carried out in the proof. The publisher wish to apologize for this oversight.

Below are the details of the corrections:

1. Page 6, Line 8 from bottom: Change "Theorem 3" to "Theorem 4".

2. Page 8, Line 11: Change "Theorem 5" to "Theorem 6".

3. Page 8, Line 12: Change "Theorems 3 and 4" to "Theorems 4 and 5".

4. Page 12, Line 9 from bottom: Use italics for the line before Section 4 .

5. Page 12, Line 7 from bottom: Change "Theorem 1" to "Theorem 2".

6. Page 13, Line 1: Change "Theorem 2" to "Theorem 3".

7. Page 13, Line 8 from bottom: Change "Theorem 3" to "Theorem 4".

8. Page 14, Line 4: Change "Theorem 3" to "Theorem 4".

9. Page 14, Line 5: Change "Theorem 4" to "Theorem 5".

10. Page 14, Line 8: Change "Theorem 5" to "Theorem 6".

11. Page 14, Line 11 from bottom: Change "Theorem 6" to "Theorem 7".

12. Page 14, Line 6 from bottom: Change "Theorem 5" to "Theorem 6".

13. Page 14, Line 4 from bottom: Change "Theorem 7" to "Theorem 8".

14. Page 15, Line 3: Change "Theorem 5" to "Theorem 6".

15. Page 15, Line 7 from bottom: Change "Theorem 8" to "Theorem 9".

16. Page 16, Line 10: Change "Theorem 5" to "Theorem 6".

17. Page 17, Line 12: Change "Theorem 3" to "Theorem 4".

The original article has been corrected. 
The online version of the original article can be found at https://doi.org/10.1007/s13171-019-00196-x

Gwo Dong Lin

Social and Data Science Research

Center, Hwa-Kang Xing-Ye

Foundation, Taipei 10659, Taiwan

E-MAIL: GDLIN@STAT.SINICA.EDU.TW

Gwo Dong Lin

Institute of Statistical Science,

Academia Sinica, Taipei, 11529 ,

TAIWAN

Chin-Yuan Hu

Department of Business Education, National Changhua University of

Education, Changhua, 50058,

TAIWAN

E-MAIL:BUHUUA@GMAIL.COM 